\title{
A terapêutica de um "CAPS AD" em um coletivo de fotografia
}

\author{
Virgínia Lima dos Santos Levy ${ }^{\text {Orcid, } \star}$ \\ Universidade Federal de Santa Catarina, Florianópolis, SC, Brasil
}

Resumo

\begin{abstract}
Sendo fundamental a manutenção de um processo de reflexão constante para que a consolidação da Reforma Psiquiátrica possa se dar sem um "engessamento" de práticas que atrapalharia a proposta de atendimento personalizado, adequado à realidade especifica de cada caso, faz-se importante observar as experiências que vêm acontecendo nos dispositivos de Atenção Psicossocial. Por isto, este artigo relata experiências ocorridas em um CAPS AD nos anos de 2011 a 2014, narrando o processo de implementação de uma oficina de fotografia e suas implicações. Percebeu-se que, propondo uma atividade aberta, flexível às demandas dos usuários, foi possível despertar interesses artísticos e de gestão, que culminaram no engajamento em um coletivo de fotografia algo que não se deu por uma obrigatoriedade de frequência a atividades, mas por uma abertura da oficina aos interesses de cada participante, o que funcionou melhor para o aumento da "adesão".
\end{abstract}

Palavras-chave: atenção psicossocial; álcool/drogas; expressão artística; fotografia; vínculo terapêutico.

\section{The Therapeutics of a “CAPS AD” in a Photography's Collective}

\begin{abstract}
Considering that it's fundamental to maintain a process of constant reflection so that the consolidation of the Brazilian Psychiatric Reform can take place without a "plastering" of practices that would hinder the proposal of personalized care, adequate to the specific reality of each case, it is important to observe the experiences that have been happening in the devices of Psychosocial Attention. For this reason, this article reports the experiences that occurred in a CAPS AD in the years 2011 to 2014, narrating the process of implantation of a photography workshop and its implications. It was noticed that, by proposing an open, flexible activity to the user's demands, it was possible to arouse artistic and management interests, which culminated in the engagement in a photography collective - something that was not due to an obligation to attend activities, but to an opening of the workshop to the interests of each participant, which worked best for increase "membership" and the frequency.
\end{abstract}

Keywords: psychosocial care; alcohol/drugs; artistic expression; photography; therapeutic link.

\section{Introdução}

Com a Reforma Psiquiátrica (BRASIL, 2005), que, após movimentos e debates anteriores (descritos por AMARANTE, 2007), tem como marco a Lei $\mathrm{n}^{\circ} 10.216$ (BRASIL, 2001), seguida pela Portaria $n^{0} 336 / G M$ (BRASIL, 2002), temos uma reformulação da assistência à pessoa com "transtornos mentais". Neste contexto, de preconização de formas não asilares de cuidado e de fomento de tentativas de reinserção comunitária e busca de autonomia, pautado pela criação de vínculos de confiança e abertura entre profissionais e sujeitos atendidos, segundo orientação do Ministério da Saúde, ${ }^{1}$ não cabe a hierarquização médico-paciente ou religioso-paciente, presente nas formas anteriores da Psiquiatria Clássica e das instituições de cunho ideológico-religioso, como comunidades terapêuticas evangelizadoras e grupos de mútua-ajuda. Para uma cogestão do cuidado, que respeita o usuário $^{2}$ dos serviços de saúde e o incentiva a conquistar direitos, faz-se necessário evitar a rigidez institucional

\footnotetext{
^Endereço para correspondência: Universidade Federal de Santa Catarina, Centro de Filosofia e Ciências Humanas, Programa de Pós-Graduação Interdisciplinar em Ciências Humanas - PPGICH - Anexo do Bloco E do CFH, 2 andar. Campus Universitário - Trindade - CEP 88.040-900 - Florianópolis, Santa Catarina - Brasil.E-mail: virginialevy@gmail.com

No ano de 2014, o Ministério da Saúde propôs, com o auxílio de equipe da Universidade Federal de Santa Catarina, o curso de capacitação "Drogas: da Coerção à Coesão", para profissionais que atuam com a clientela AD. O texto de Barreiros é parte do $4^{\circ}$ módulo deste curso (unidade 2).

${ }^{2}$ No caso específico da unidade de saúde aqui citada, é importante observar que, embora exista na lei a substituição de "pacientes" por "usuários" para evita hierarquizações e formas pejorativas de tratamento, esta substituição não é bem vista, posto que o termo também se refere a "usuários de drogas", utilizado muitas vezes de forma também pejorativa. Por este motivo, e também pela força da cultura hospitalocêntrica, os usuários desta unidade demonstram preferência por ser denominados "pacientes".
}

explicitada por Barreiros (2014), rigidez ainda incentivada no caso do cuidado às pessoas com transtornos relacionados ao uso abusivo de substâncias psicoativas. Tal incentivo se deve à concepção antiga de que "falhas de caráter" seriam responsáveis pelos "vícios", devendo o profissional ser mais rígido com usuários do que seria em sua vida cotidiana, para efetivar um "tratamento moral". Na prática profissional, contudo, vemos que estas tentativas de "exclusão social" e "normatização dos corpos" têm efeito negativo, funcionando ora de forma iatrogênica, ora como barreiras ao acesso do usuário ao cuidado em Saúde Mental.

Dentro desta perspectiva, torna-se relevante pensar e repensar constantemente as práticas terapêuticas dos novos dispositivos da Atenção Psicossocial, evitando que o "engessamento" de posturas e atividades possa tornar as ofertas menos "acolhedoras", menos adequadas à necessidade real e individual de cada atendido. Por conta disto, propõe-se, neste artigo, uma reflexão sobre a criação e a implantação de oficinas terapêuticas, através do percurso de uma oficina específica (a Oficina de Fotografia), com enfoque sobre as experiências ocorridas no período entre 2011 e 2014. A proposta, neste trabalho, é pensar sobre as relações entre oficinas artísticas, oferta de cuidado e fomento da autoestima e da reinserção social de usuários de um CAPS.

\section{Grupo Phoenix - da implantação de uma oficina ao surgimento de um coletivo artístico}

Dado o caráter ainda recente das transformações estruturais da Política Nacional de Saúde Mental, é ainda comum, nos pontos de atenção da Rede de Atenção Psi- 
cossocial (RAPS), a presença de profissionais que, sem muitas discussões sobre Saúde Coletiva e Saúde Mental durante a formação, vão "aprender na prática" pontos teóricos, alguns dos quais já consagrados, além de, infelizmente, repetir concepções e práticas errôneas enraizadas nos serviços. Zurba (2012), por exemplo, descreve o processo de modificação do currículo do curso de graduação em Psicologia da UFSC, a partir de 2008. Esta modificação ocorre no sentido de aproximar mais o curso da participação do psicólogo nas Políticas Públicas, incluindo questões que passavam ao largo da formação voltada para a prática profissional em consultório particular. Se mesmo na Psicologia (voltada para as questões de Saúde Mental) esta reformulação curricular é (ainda) nova, o mesmo (ou pior) acontece na formação dos outros profissionais de saúde que compõem a equipe dos CAPS. Neste sentido, é compreensível que, no final de 2011, um trabalho pudesse começar com profissionais com pouca bagagem teórica sobre saúde mental e oficinas terapêuticas. Assim, foi na experiência de implantação de oficinas, antes que se decidisse prosseguir com estudos acadêmicos, que se percebeu que, para que o trabalho em um CAPS AD fosse possível, seria necessário mais do que o oferecimento de diversas atividades descritas na Política Nacional de Saúde Mental. Para a viabilização de Projeto Terapêutico Singular efetivamente individualizado, não é suficiente a oferta variada de atividades terapêuticas: é preciso que as práticas de cuidado "contemplem a flexibilidade e a abrangência possíveis e necessárias a esta atenção específica", como aponta o Ministério da Saúde (BRASIL, 2003).

Seguindo esta lógica, é fácil compreender como outras oficinas precisaram se adaptar aos interesses e possibilidades dos usuários para funcionar. E é dentro deste esforço de adaptação que surge, no final de 2011, a proposta de uma Oficina de Fotografia, implantada em conjunto com a farmacêutica da Unidade. Embora esta profissional possua formação em Fotografia, e a psicóloga (autora deste trabalho) possua experiência anterior com produção de vídeos, é importante frisar que não se deve apenas aos interesses das profissionais, e sim ao interesse verbalizado por alguns usuários, a escolha desta oficina, que vem ainda para atender à demanda de alguma atividade que se realizasse no final da tarde, horário em que havia poucas atividades, embora fosse um horário em que, dada a proximidade da noite e do horário de fechamento do CAPS, houvesse um relatado aumento dos níveis de ansiedade pela clientela ali atendida. Além do pedido de trabalho com fotografia, havia, portanto, um pedido de "grupo de atendimento de emergência", que ofertasse algum tipo de escuta em menos tempo àqueles que necessitassem de acolhimento antes de voltar para casa - em menos tempo do que teriam que aguardar para um atendimento com seus técnicos de referência.

Diante dessas demandas, pensou-se que elas poderiam ser atendidas ao mesmo tempo, em um grupo de uma hora de duração, nas quartas-feiras (horário logo ampliado conforme a expansão dos interesses dos usuários), que teria como base a ideia de trabalhar as relações entre imagens, símbolos e percepções de realidade, assim como a observação das transformações "sofridas" pelo papel fotográfico após processos físicos e químicos propositais e não propositais.

Ao contrário do ocorrido na Oficina de "Cinema" descrita por Levy (2016), que se inicia com um cronograma preestabelecido de atividades (do qual a oficina se desfaz para atender aos participantes), a Oficina de Fotografia não se inicia com passos estritamente definidos, mas com um guia geral de atividades. A cada encontro haveria ensino e utilização de técnicas de trabalho fotográfico, a partir de um espaço inicial de fala sobre questões do cotidiano e interesses dos participantes (o que os levou a estar na oficina naquele momento e o que pensam que podemos fazer naquele encontro). Assim, a realização de fotografias não é, primeiramente, o foco principal da oficina, e sim um disparador de uma convivência agradável que tem efeitos benéficos, em consonância com o descrito por Menezes, Teixeira e Yasui (2008) a respeito de uma oficina de fotografia em um CAPS de Tocantins.

Ainda assim, a atividade em si tem a sua relevância aumentada pelo interesse progressivo dos usuários. Mesmo que a oficina continue aberta (ou seja, receba todos os participantes que desejem, ainda que não tenham sido indicados por seus técnicos de referência), de frequência flutuante, um grupo de usuários começa a ter frequência regular, interessando-se pela gestão deste espaço terapêutico. Este grupo, é importante frisar, não é formado pelas pessoas que têm a oficina como atividade obrigatória de seu plano de tratamento, mas pelas pessoas que, não o tendo, constroem vínculos com a oficina. Tornam-se mais comuns propostas como saídas fotográficas temáticas (visitas ao "Museu do Índio" e à "Praia de Botafogo", por exemplo) e não temáticas (passeios pelas redondezas), e o grupo se torna coeso, constituindo um coletivo artístico. Após votação, ficou definido que o grupo se chamaria "Grupo Phoenix - Coletivo de Fotografia" (em alusão ao mito da Fênix, pássaro capaz de renascer das próprias cinzas), e que deveria haver a exposição dos trabalhos e assinatura dos autores. ${ }^{3}$

Após um ano de oficina (inicialmente proposta para o período de novembro de 2011 a março de 2012) e da criação do logotipo do grupo, tivemos a primeira exposição de trabalhos, realizada dentro do próprio CAPS. Foi escolhido, em votação, o título "Recuperação em FOCO", inserido sobre a fotografia, feita por uma participante de si própria ao espelho, para fazer a divulgação da exposição (de 17/12/2012 a 04/01/2013) e da abertura do evento, na manhã do dia 17. São impressas fotografias feitas por todos os participantes da oficina (entre usuários e técnicos), e coladas sobre placas de acrílico penduradas na unidade, de modo que pudessem ser vistas por todos, e não apenas por aqueles que as procurassem. Apesar do desejo dos membros do coletivo de que a exposição pu${ }^{3}$ Alguns usuários deste CAPS AD preferem se manter anônimos, utilizando siglas e pseudônimos para evitar preconceitos por se verem ligados a um CAPS AD (e, portanto, serem vistos como "drogados"). Mesmo nestes casos, porém, foi preferível valorizar a singularidade dos sujeitos e de suas construções artísticas, a exemplo do que aconteceu nas pinturas dos pacientes de Nise da Silveira e a despeito do que ocorre com os trabalhos em alguns CAPS. Para conhecer o trabalho de Nise, além de seus livros, como "O Mundo das Imagens" e "Imagens do Inconsciente", uma possibilidade é a tese de Sandra Fernandes (2015), listada nas referências deste artigo. 
desse "incomodar", fazer deslocar do habitual, rotineiro (como é tarefa da arte e da psicologia), há algumas críticas por parte da equipe administrativa de que aquele é um ambiente de saúde, não podendo ser perturbado por atividades artísticas (como uma peça de acrílico atrapalhando o caminho em um corredor estreito). Destaco esta crítica não para desqualificar a equipe, mas para trazer à discussão a questão do lugar da arte nos espaços da Saúde Mental: seria o de entretenimento? O de expressão? O de experimentação do lúdico? $\mathrm{O}$ de novas possibilidades de atuação no mundo? O de profissionalização? Mesmo com a já clássica relação entre arte e trabalho em Saúde Mental (descrita na obra de Nise da Silveira, entre outras), o lugar da arte ainda parece, em muitos momentos, ser subalterno ao das práticas biomédicas.

Este questionamento não implica, entretanto, em desprezar a importância da fotografia como ferramenta clínica, descrita por autores como Mattos, Zanella e Nuernberg (2014), Freitas (2012), e mesmo Neiva-Silva e Koller (2002), dentre outros. Como bem observa Ana Freitas (2012, p. 21), a fotografia "possibilita a escuta do singular e a constituição de um sujeito desejante, autônomo, inserido no social", razão pela qual pode constituir um potente dispositivo de cuidado, se implantada conforme esta perspectiva. Neste sentido, Levy (2015) aponta para a necessidade de que o trabalho em Saúde Mental, principalmente na área dos transtornos relativos ao uso abusivo de álcool/outras drogas, deve ser o de ampliação do leque de possibilidades de ser/estar no mundo, de aumento da possibilidade de vislumbrar, criar e buscar atuar conforme projetos de vida. Isto se mostrou possível na Oficina de Fotografia, em que um grupo de pessoas passa a se identificar como grupo unido não pela carga pejorativa do consumo anterior de substâncias psicoativas ou pelas dificuldades atuais no "tratamento" relacionado a este consumo, mas como artistas fotógrafos - ou ainda, como fotógrafos mais "comerciais", uma vez que passam a ser frequentemente convocados a assumir o papel de registro das atividades do CAPS não promovidas pelo coletivo de fotografia, como festas de natal e passeios.

Com o sucesso das exposições internas e a vontade dos participantes de outras oficinas da unidade de expor trabalhos, foi crescente a motivação para procurar um espaço para exposições externas, concretizado no final de 2012, e a participação em exposições organizadas por outras instituições, como a Coordenação Municipal de Saúde Mental e a Mostra de Arte Insensata, desenvolvida em Minas Gerais e tornada presente em diversos estados. Ainda que não concretizado, é importante destacar o desejo de apresentação em mostras artísticas que não sejam da Saúde Mental, ressaltando-se o potencial dos participantes de produção de trabalhos de considerável valor artístico.

\section{Considerações finais}

A exemplo do que havia sido descrito anteriormente em outros trabalhos, como o de Menezes, Teixeira e Yasui (2008), também no caso específico desta oficina de fotografia foi possível perceber que, na terapêutica em Saúde Mental na perspectiva da Atenção Psicosso- cial, é importante que o foco inicial dos grupos seja sobre os participantes, e não sobre as tarefas. Ainda que seja interessante que o participante possa, a longo prazo, descobrir atividades com que se identifique, esta "identificação" só é possível se a atividade lhe ajuda no processo constante de constituição de si mesmo enquanto sujeito, a partir da produção de sentido sobre a própria história de vida. Não está no ato em si de fotografar, assim como em qualquer outro ato (como o de fazer desenhos, pinturas, trabalhos manuais, escrita, esportes, etc.), o que torna a atividade intrinsecamente terapêutica, mesmo que o uso da arte como forma de expressão torne mais possível que o trabalho com as questões psíquicas seja efetuado.

Para que a oficina de fotografia pudesse ser, de fato, útil aos seus participantes, foi necessário deixar de lado a rigidez institucional típica destes espaços, flexibilizando os horários e as escolhas de atividades. Na sexta-feira anterior às exposições, por exemplo, foi comum que o coletivo (participantes usuários e técnicos) estivesse na unidade em horário que ia além do horário de funcionamento normal da unidade, e, em muitos momentos, a oficina funcionou por um horário muito além daquele que era o programado. Esta "plasticidade na oferta do cuidado" de que fala Barreiros (2014, p. 51) foi muito importante tanto para a construção de vínculos que permanecem mesmo após a saída de alguns participantes da Unidade quanto para a valorização dos sujeitos enquanto cidadãos "autônomos", capazes de gerir suas vidas conforme seus sonhos, valores e interesses - o que constitui o foco da Atenção Psicossocial.

\section{Referências}

AMARANTE, P. Saúde Mental e Atenção Psicossocial. Rio de Janeiro: FIOCRUZ, 2007.

BARREIROS, G. B. Construindo e consolidando o vínculo. In: BÜCHELE, F.; PETUCO, D. R. S. (Org.). Organização dos serviços para garantir acesso e promover vinculação do usuário de drogas [online]. Florianópolis: Departamento de Saúde Pública/UFSC, 2014. Disponível em: https://ares.unasus. gov.br/acervo/handle/ARES/1836. Acesso em: 10 jul. 2014.

BRASIL. Presidência da República. Casa Civil. Subchefia para Assuntos Jurídicos. Lei $n^{\circ}$. 10.216, de 6 de abril de 2001. Dispõe sobre a proteção e os direitos das pessoas portadoras de transtornos mentais e redireciona o modelo assistencial em saúde mental. Disponível em: http://www.planalto.gov.br/ ccivil_03/Leis/LEIS_2001/L10216.htm. Acesso em: 19 dez. 2015.

BRASIL. Ministério da Saúde. Secretaria de Assistência à Saúde. Portaria GM/MS $n^{\circ}$ 336, de 19 de fevereiro de 2002. Estabelece as modalidades de serviços dos Centros de Atenção Psicossocial. Disponível em: http://bvsms.saude.gov.br/bvs/ saudelegis/gm/2002/prt0336_19_02_2002.html. Acesso em: 10 jan. 2015.

BRASIL. Ministério da Saúde. Secretaria Executiva. Coordenação Nacional de DST e AIDS. A politica do Ministério da Saúde para a atenção integral a usuários de álcool e outras drogas. 2003. Disponível em: http://bvsms.saude.gov.br/bvs/ publicacoes/politica_atencao_alcool_drogas.pdf. Acesso em: 18 jan. 2018.

Fractal, Rev. Psicol., v. 30 - n. 3, p. 310-313, 2018 
BRASIL. Ministério da Saúde. Secretaria de Atenção à Saúde. DAPE. Coordenação Geral de Saúde Mental. Reforma Psiquiátrica e Política de Saúde Mental no Brasil: Documento apresentado à Conferência Regional de Reforma dos Serviços de Saúde Mental: 15 anos depois de Caracas. OPAS. Brasília: MS, 2005. Disponível em: http://bvsms.saude.gov.br/bvs/ publicacoes/Relatorio15_anos_Caracas.pdf. Acesso em: 10 jan. 2018.

FERNANDES, S. Nise da Silveira e a saúde mental no Brasil: um itinerário de resistência. 2015. 206 f. Tese (Doutorado)ProgramadePós-GraduaçãoemCiências Sociais daUniversidade Federal do Rio Grande do Norte, Natal, 2015. Disponível em: https://repositorio.ufrn.br/jspui/bitstream/123456789/21426/1/ SandraMichelleBessaDeAndradeFernandes_TESE.pdf. Acesso em: 20 abr. 2018.

FREITAS, A. A fotografia como recurso terapêutico em um CAPS: a possibilidade de novos olhares. 2012. $35 \mathrm{f}$. Trabalho de Conclusão de Curso (Residência Integrada em Saúde) Grupo Hospitalar Conceição - GHC/RS, Porto Alegre, 2012. Disponível em: http://coleciona-sus.bvs.br/lildbi/docsonline/ get.php?id=507. Acesso em: 2 abr. 2018.

LEVY, V. Narrativas de usuários de crack: o dizer sobre si e o mundo através do audiovisual. 2015. 105 f. Dissertação (Mestrado Profissional)-Programa de Pós-Graduação em Saúde Mental e Atenção Psicossocial da Universidade Federal de Santa Catarina, Florianópolis, 2015. Disponível em: http:/ tede.ufsc.br/teses/PPSM0022-D.pdf. Acesso em: 24 out. 2016.

LEVY, V. Oficinas terapêuticas e produção de vínculo em CAPS AD. Cadernos Brasileiros de Saúde Mental, Florianópolis, v. 8, n. 19, p. 97-106, 2016. Disponível em: http://incubadora. periodicos.ufsc.br/index.php/cbsm/article/view/3364/4672. Acesso em: 11 dez. 2016.

MATTOS, L.; ZANELLA, A.; NUERNBERG, A. Entre olhares e (in)visibilidades: reflexões sobre fotografia como produção dialógica. Fractal: Revista de Psicologia, Niterói, v. 26, n. 3, p. 901-918, dez. 2014. Cross ${ }^{\text {Ref. }}$

MENEZES, M.; TEIXEIRA, I.; YASUI, S. O olhar fotográfico como proposta de cuidado em saúde mental. Arquivos Brasileiros de Psicologia, Rio de Janeiro, v. 60, n. 3, 2008. Disponível em: http://seer.psicologia.ufrj.br/index.php/abp/ article/view/290/247. Acesso em: 2 abr. 2018.

NEIVA-SILVA, L.; KOLLER, S. O uso da fotografia na pesquisa em Psicologia. Estudos de Psicologia, Natal, v. 7, n. 2, jul./dez. 2002. Cross ${ }^{\text {Ref. }}$

ZURBA, M. do C. (Org.). Introdução. In: Psicologia e Saúde Coletiva. Florianópolis: Tribo da Ilha, 2012. p. 21-24. Disponível em: http://psicologia.paginas.ufsc.br/files/2012/06/ Miolo_Psicologia-e-Saude.pdf. Acesso em: 12 maio 2018.

Recebido em: 14 de julho de 2015

Aceito em: 13 de abril de 2018 